\title{
Adipose-derived stem cells improve the viability of nucleus pulposus cells in degenerated intervertebral discs
}

\author{
KERAN SONG ${ }^{1 *}$, TAO GU ${ }^{2 *}$, FENG SHUANG ${ }^{3}$, JIAGUANG TANG $^{1}$, \\ DONGFENG REN ${ }^{1}$, JIANG QIN ${ }^{1}$ and SHUXUN HOU ${ }^{1}$ \\ ${ }^{1}$ Institute of Orthopaedics, The First Affiliated Hospital of Chinese PLA General Hospital; \\ ${ }^{2}$ Department of Orthopedic Surgery, Navy General Hospital, Beijing 100048; ${ }^{3}$ Department of Orthopedic Surgery, \\ The $94^{\text {th }}$ Hospital of Chinese PLA, Nanchang, Jiangxi 330002, P.R. China
}

Received August 3, 2014; Accepted April 15, 2015

DOI: $10.3892 / \mathrm{mmr} .2015 .3895$

\begin{abstract}
Patients with degenerative disc disease (DDD) experience serious clinical symptoms, including chronic low back pain. A series of therapies have been developed to treat DDD, including physical therapy and surgical treatment. However, the therapeutic effect of such treatments has remained insufficient. Recently, stem cell-based therapy, in which stem cells are injected into the nucleus pulposus in degenerated intervertebral disc tissue, has appeared to be effective in the treatment of DDD. In the present study, the effect of adipose-derived stem cells on degenerated nucleus pulposus cells was investigated using a co-culture system to evaluate the biological activity of degenerated nucleus pulposus cells. Human degenerated nucleus pulposus tissue was obtained from surgical specimens and the adipose-derived stem cells were derived from adipose tissue. The degenerated nucleus pulposus cells were cultured in a mono-culture or in a co-culture with adipose-derived stem cells using $0.4-\mu \mathrm{m}$ Transwell inserts. The results indicated that adipose-derived stem cells were able to stimulate matrix synthesis and the cell proliferation of degenerated nucleus pulposus cells, promoting the restoration of nucleus pulposus cells in the degenerated intervertebral disc.
\end{abstract}

\section{Introduction}

Diseases of the lumbar intervertebral disc (IVD) affect the majority of the adult population, causing back pain, sciatica, disc herniation and spinal stenosis, in addition to other distressing and disabling spinal symptoms (1-3). The IVD contains two

Correspondence to: Professor Shuxun Hou, Institute of Orthopaedics, The First Affiliated Hospital of Chinese PLA General Hospital, 51 Fucheng Road, Haidian, Beijing 100048, P.R. China E-mail: shuxunhou0517@126.com

*Contributed equally

Key words: interaction, nucleus pulposus cells, adipose-derived stem cells, co-culture, proliferation sections: The annulus fibrosus (AF) and the nucleus pulposus (NP) (4). The AF is a ring of flexible fibrocartilage, which surrounds the soft center consisting of the NP. The IVD acts as a shock buffer when the spine encounters compression (5). It has been reported that IVD degeneration initiates in the NP with cellular loss, proteoglycan content breakdown and decreased water-binding capacity (6).

Current therapies for the treatment of IVD disease include non-invasive and invasive therapies, which are able to relieve the symptoms, but not resolve the condition completely $(7,8)$. Methods for increasing the proliferative capacity and matrix synthesis of NP cells (NPCs) include gene therapy and cell therapy $(9,10)$. Among them, regenerative medicine based on cell therapy is one of the most promising approaches in the treatment of IVD disease (11). Gruber et al (12) attempted to use autologous disc cell implantation to treat the disc degeneration in a sand rat model. Okuma et al (13) reported that the re-insertion of stimulated NPCs retards IVD degeneration. However, autologous disc cell transplantation is difficult, as it is, at present, not possible to harvest a high quantity of autologous disc cells from a single IVD, which would cause damage to the donor site. Recent advances in cellular and molecular biology indicated that stem cells exhibit great potential to be applied in cell therapy $(14,15)$. Stem cells are able to differentiate into different types of cells, including osteoblasts, chondrocytes, adipocytes, cardiomyocytes, hepatocytes and epithelial cells (16). By contrast, stem cells secrete a large quantity of growth factors, which are vital in cell therapy (17-19). A number of studies have suggested that mesenchymal stem cells (MSCs) may be used in the treatment of IVD diseases (20). Yamamoto et al (21) observed that bone marrow-derived stromal cells may improve the viability of NPCs and direct cell-to-cell contact in a co-culture system between NPCs and bone marrow MSCs (BMSCs), accomplishing a significant increase in the viability of NPCs. Wei et al (22) demonstrated that rodent BMSCs were able to differentiate into IVD-like cells following co-culture with rat disc tissue. Sun et al (23) noted that ADSCs protect compressive load-induced NP cell death and degradation by inhibition of activated caspase- 9 and -3 activity in the co-culture system.

Therefore, in the present study, the effect of adipose-derived stem cells (ADSCs) on degenerated NPCs was investigated using a co-culture system, and the viability of NPCs following 
co-culture with BMSCs was determined, in order to evaluate the potential application of BMSCs in the treatment of IVD diseases.

\section{Materials and methods}

The present study was approved by the Institutional Review Board of the General Hospital of Chinese People's Liberation Army (Beijing, China) and informed consent was obtained from all patients.

Cell isolation and culture. The human adipose tissue was harvested from patients who had undergone lipoaspirate procedures. To harvest ADSCs, the obtained tissue was washed with phosphate-buffered saline (Gibco Life Technologies, Carlsbad, CA, USA) to remove red blood cells, cut into small sections and then digested with $0.075 \%$ collagenase (NB4; SERVA Electrophoresis $\mathrm{GmbH}$, Heidelberg, Germany) for $60 \mathrm{~min}$ at $37^{\circ} \mathrm{C}$. The digested tissues were filtered using a $200-\mu \mathrm{m}$ filter to remove tissue debris and obtain a single-cell suspension. The cell suspension was centrifuged at $250 \mathrm{x}$ g for $10 \mathrm{~min}$, and the pellet was then re-suspended in Dulbecco's modified Eagle's medium containing $10 \%(\mathrm{v} / \mathrm{v})$ fetal bovine serum (Hyclone, Logan, UT, USA). The cells were seeded in 100-mm tissue culture dishes at a density of $2 \times 10^{4}$ cells $/ \mathrm{cm}^{2}$ and cultured at $37^{\circ} \mathrm{C}$ in a humidified incubator with $5 \% \mathrm{CO}_{2}$. After 7-10 days, adherent cells were trypsinized (Gibco Life Technologies) and sub-cultured as described previously (24).

Degenerated NPCs were harvested from NP tissues, which were obtained from the DDD patients undergoing surgical procedures as described previously (24). NPCs at passage 2 were used in the present study.

Groups. A six-well culture plate $\left(\operatorname{Costar}^{\circledR}\right.$, Corning, Inc., Corning, NY, USA) and Transwell ${ }^{\circledR}$ inserts (Corning, Inc.) were used for the co-culture. The Transwell insert used in the present study consisted of a polyethylene terephthalate track-etched membrane with $0.4-\mu \mathrm{m}$ pores at the bottom, which prevented cell migration. The study groups consisted of the control group (NPCs seeded onto the Transwell) and the experimental group (NPCs seeded onto the Transwell and ADSCs seeded onto a six-well plate).

A total of $1 \times 10^{4}$ NPCs or ADSCs at passage 2 were seeded in each group. The medium was changed every 2 days.

Cell proliferation. Cell proliferation was determined using the Cell Counting Kit-8 (CCK-8; Dojindo Laboratories, Kumamoto, Japan). A total of $10^{3}$ NPCs from the two groups in $100 \mu 1$ were seeded into every well of the 96-well plate. After 12 h, $10 \mu \mathrm{l}$ CCK-8 solution was added into each well. After a further $4 \mathrm{~h}$, the absorbance of the supernatant was measured spectrophotometrically at $450 \mathrm{~nm}$ (Evolution $^{\mathrm{TM}} 201$ UV-visible Spectrophotometer; Thermo Fisher Scientific, Waltham, MA, USA). The level of cell proliferation at days 1, 3, 5 and 7 was assessed. Cell counts were determined using a calibration curve.

DNA and glycosaminoglycan (GAG) synthesis. The DNA content was quantified using a Qubit dsDNA HS assay kit (Invitrogen Life Technologies, Carlsbad, CA, USA) following
Table I. Primer sequences for reverse transcription-quantitative polymerase chain reaction.

\begin{tabular}{llc}
$\begin{array}{l}\text { Gene } \\
\text { symbol }\end{array}$ & \multicolumn{1}{c}{ Primer } & $\begin{array}{c}\text { Product } \\
(\mathrm{bp})\end{array}$ \\
\hline $\begin{array}{l}\text { Type II } \\
\text { Collagen }\end{array}$ & F, 5'-CGAAAGGTCAGACGGGTGAA-3' & 373 \\
Aggrecan & F, 5'-ACCTCACCATGCCTTCACTG-3' & 316 \\
& R, 5'-GCTCTCACCTTTCACCACGA-3' & \\
Sox-9 & F, 5'-AGGAGAACCCCAAGATGCAC-3' & 110 \\
& R, 5'-GAGGCGTTTTGCTTCGTCAA-3' & \\
GAPDH & F, 5'-CCACATCGCTGAGACACCAT-3' & 353 \\
& R, 5'-AAATGAGCCCCAGCCTTCTC-3' & \\
\hline
\end{tabular}

Sox-9, sex determining region Y-box 9; F, forward; R, reverse.

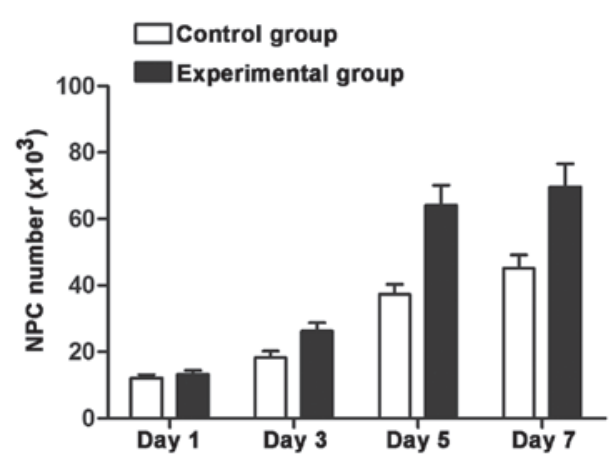

Figure 1. Proliferation of nucleus pulposus cells. The NPCs proliferated gradually in the two groups with increasing culture time. No significant difference was identified between the two groups on the first day $(\mathrm{P}>0.05)$. However, a significant difference was observed on days 3,5 and $7(\mathrm{P}<0.05)$. Values are expressed as the mean \pm standard deviation. NPC, nucleus pulposus cell.

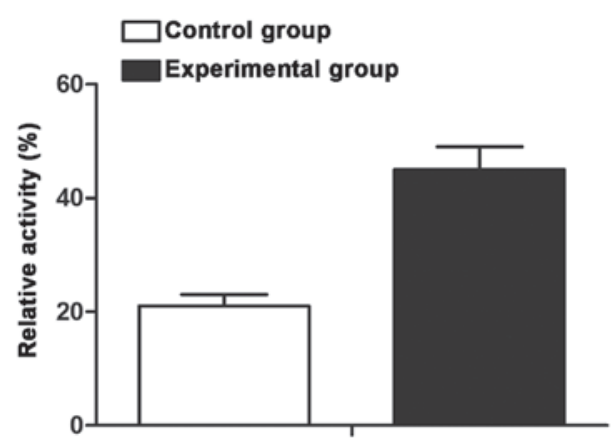

Figure 2. Telomerase activity of nucleus pulposus cells. Telomerase activity of nucleus pulposus cells in the co-culture group was higher than that in the nucleus pulposus cell mono-culture group $(\mathrm{P}<0.05)$. Values are expressed as the mean \pm standard deviation.

the manufacturer's instructions. The DNA content was quantified using the Qubit dsDNA HS assay kit (cat. no. Q32854, Life Technologies, Grand Island, NY, USA) and the Qubit 2.0 Fluorometer (Life Technologies) according to the manufacturer's instructions. Briefly, $1 \mu \mathrm{l}$ DNA sample at $5 \mathrm{ng} / \mu \mathrm{l}$ was diluted 200-fold in Qubit dsDNA HS buffer in clear plastic Qubit assay 
A

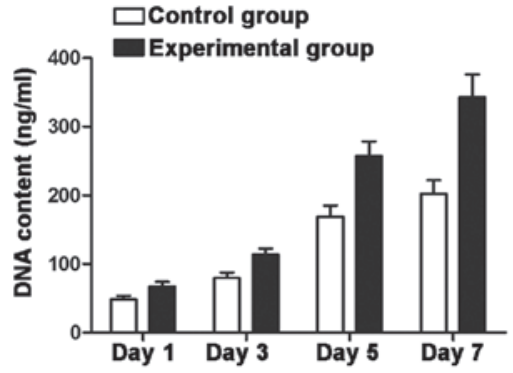

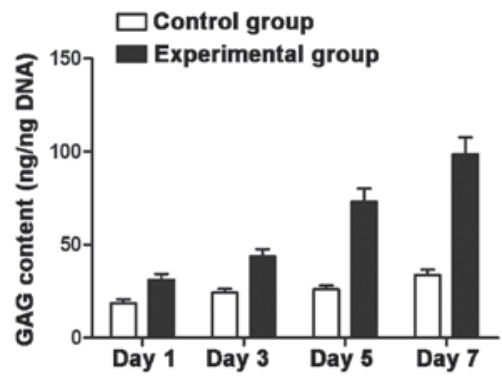

Figure 3. (A) DNA content and (B) GAG synthesis. Results regarding the DNA content were consistent with the proliferation. GAG content of NPCs in the co-culture group was higher than that in the NPC mono-culture group $(\mathrm{P}<0.05)$. Values are expressed as the mean \pm standard deviation. NPC, nucleus pulposus cells; GAG, glycosaminoglycan.
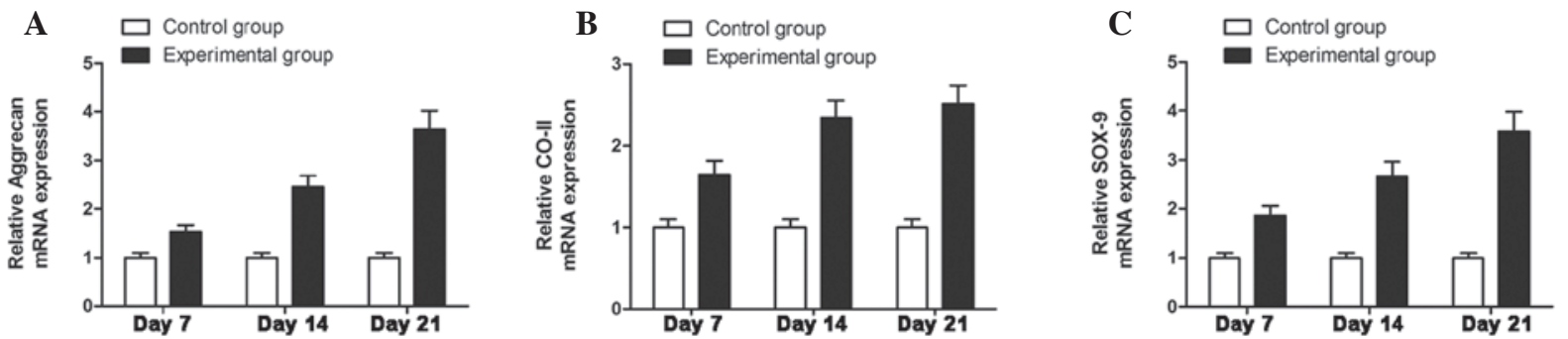

Figure 4. Gene expression of NPCs. Expression of (A) aggrecan, (B) type II collagen and (C) SOX-9 in the NPCs in the co-culture group was higher than that in the NPC mono-culture group $(\mathrm{P}<0.05)$. Values are expressed as the mean \pm standard deviation. NPC, nucleus pulposus cell; SOX-9, sex determining region Y-box 9; CO-II, type II collagen.

A

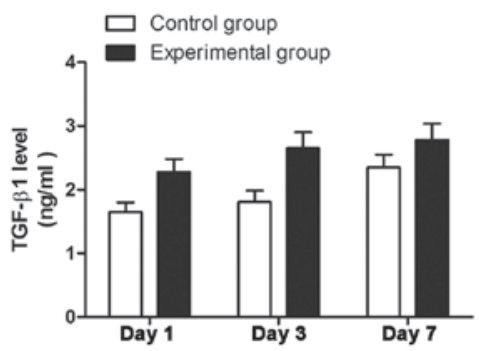

B

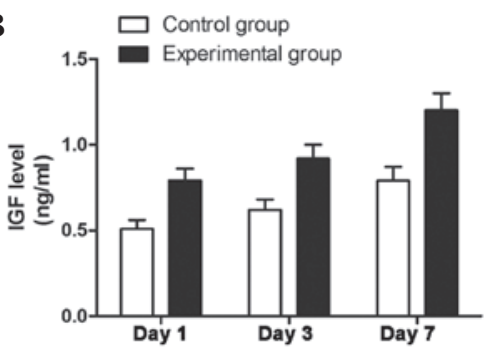

Figure 5. Protein levels of growth factors. Significant differences were identified in the levels of the growth factors (A) TGF- $\beta 1$ and (B) IGF between the co-culture group and the NPC mono-culture group $(\mathrm{P}<0.05)$. Values are expressed as the mean \pm standard deviation. NPC, nucleus pulposus cell; TGF, transforming growth factor; IGF, insulin-like growth factor.

tubes (cat. no. Q32856, Life Technologies) and measured on the fluorometer. Prior to taking the measurements, a two-point calibration curve was established using the supplied standards with the kit, at $0 \mathrm{ng} / \mu \mathrm{l}$ and $10 \mathrm{ng} / \mu \mathrm{l}$. Samples that fell below the limit of quantification of $0.5 \mathrm{ng} / \mathrm{ml}(0.1 \mathrm{ng} / \mu 1$, diluted 200 -fold $)$ were not reported by Qubit. The contents of GAG were measured using a Blyscan assay kit (Biocolor, Carrickfergus, Northern Ireland) as described previously (25).

Telomerase activity. The telomerase activity was determined using a telomerase polymerase chain reaction (PCR) ELISA kit as described previously (26). Following incubation for 7 days, the NPCs from the two groups were homogenized in a lysis buffer [(0.5\% CHAPS, $10 \mathrm{mM}$ Tris-HC1 (pH 7.5), $1 \mathrm{mM} \mathrm{MgCl}_{2}, 1 \mathrm{mM}$ EGTA, $5 \mathrm{mM} \beta$-mercaptoethanol, $0.1 \mathrm{mM}$ AEBSF and 10\% glycerol)] and centrifuged at $3,000 \mathrm{x} g$ for $10 \mathrm{~min}$. The extracts were subsequently subjected to a telomeric repeat amplification protocol. The elongated fragments were then amplified using PCR. The PCR products were detected and quantified by performing ELISA according to the manufacturer's instructions and the results were normalized to those obtained for a standard.

Differentiation characteristics of NPCs. The relative expression of sex determining region Y-box 9 (SOX-9), aggrecan and type II collagen was measured in the two groups at days 7, 14 and 21 to evaluate the differentiation characteristics of NPCs. RNA was extracted from co-cultured NPCs and control groups (NPC mono-culture) using TRIzol reagent (Invitrogen Life Technologies), according to the manufacturer's instructions, and reverse transcribed $(5 \mu \mathrm{g})$ into cDNA using BioScript ${ }^{\mathrm{TM}}$ reverse transcriptase (Bioline, Taunton, MA, USA), according to the manufacturer's instructions. Reverse transcription-quantitative PCR (RT-qPCR) was used to evaluate the expression of the cartilage-specific genes SOX-9, aggrecan and type II collagen. The primer sequences (Beijing Sunbiotech Co., Ltd., Beijing, China) are listed in Table I and the cycling parameters were as follows: Denaturation at $94^{\circ} \mathrm{C}$ for $2 \mathrm{~min}$; 40 cycles at 
$94^{\circ} \mathrm{C}$ for $15 \mathrm{sec}$ and $60^{\circ} \mathrm{C}$ (or $5^{\circ} \mathrm{C}$ below melting temperature) for $1 \mathrm{~min}$. Amplification reactions were performed in duplicate and the quantity of cDNA in the reactions was normalized with an internal control, the constitutively expressed gene GAPDH. The specificity of the amplification of the expected DNA fragments was confirmed on $2 \%$ agarose gel electrophoresis and by analysis of the melting curves. An amplification reaction control with no reverse transcriptase enzyme, termed RT-, was performed in order to assess the interference of potential genomic DNA in the RNA solution. Relative gene expression was calculated using the formula: $\triangle \mathrm{CT}=\mathrm{CT}_{\mathrm{GAPDH}}-\mathrm{CT}_{\text {target }}$.

Level of growth factors in supernatants. ELISA was used to quantitatively determine the levels of transforming growth factor (TGF)- $\beta 1$ and insulin-like growth factor (IGF) in the supernatant in the two groups at different time-points (27) using the TGF- $\beta 1$ human ELISA kit (cat. no. ab100647, Abcam, Cambridge, UK) and the IGF1 human ELISA kit (cat. no. ab100545, Abcam).

Statistical analysis. One-way analysis of variance was performed using SPSS 12.0 (SPSS, Inc., Chicago, IL, USA). If the analysis of variance indicated a significant difference $(\mathrm{P}<0.05)$ between the groups, the difference was evaluated using the least significant difference test. Values are expressed as the mean \pm standard deviation.

\section{Results}

Co-culture with ADSCs enhances proliferation of NPCs. The number of cells increased gradually in the two groups with increasing culture time. The cell yields in cultured NPCs exhibited an increase in the control and experimental groups. No significant difference was identified between the two groups on the first day; however, a significant difference appeared as the time period increased (days 3, 5 and $7 ; \mathrm{P}<0.05$ ) (Fig. 1).

ADSCs enhance telomerase activity in NPCs. NPCs cultured in the co-culture group exhibited a higher level of relative telomerase activity (44.9\%) as compared with that in NPCs in the mono-culture group (21.1\%), and a significant difference was identified between the two groups $(\mathrm{P}<0.05)$ (Fig. 2).

GAG and DNA content of NPCs are increased in co-culture with ADSCs. The results from the DNA content analysis showed a marked increase in DNA content in the co-culture group following 5 and 7 days of culture, and were therefore consistent with the results regarding the level of proliferation (Fig. 3A). Co-culturing of NPCs and ADSCs resulted in a significant increase in total GAG as compared with that in the NPC mono-culture $(\mathrm{P}<0.05)$ (Fig. 3B).

Expression of genes associated with differentiation is enhanced in NPCs in co-culture with ADSCs. The gene expression of type II collagen, aggrecan and SOX-9 reflects the chondrogenic differentiation of NPCs. Fig. 4 demonstrates that the expression of type II collagen, aggrecan and SOX-9 was upregulated with increased culture time. Compared with the mRNA levels in NPC mono-culture, the NPCs in the co-culture groups exhibited a significantly higher gene expression $(\mathrm{P}<0.05)$.
ADSCs secrete growth factors during co-culture with NPCs. The levels of TGF- $\beta 1$ and IGF-1 in the supernatant in the co-culture group was higher than that in the NPC mono-culture group, which indicated that ADSCs secrete growth factors during the co-culture process.

\section{Discussion}

Stem cell-based cell therapy provides a novel promising treatment for IVD disorders. In previous years, there have been an increasing number of studies focusing on the treatment of IVD disorders, particularly those using BMSCs. BMSCs have been observed to have the capability of differentiating into mesenchymal tissues, including chondrocytes, adipocytes, osteoblasts, hepatocytes and epithelial cells, among others $(28,29)$. In addition, BMSCs may secrete growth factors, which would feed or nurse other cells. Certain studies have demonstrated that the restoration of BMSCs may improve IVD degeneration (30). Therefore, in the present study, the improvement of the biological and metabolic viability of degenerated NPCs following co-culture with BMSCs in a co-culture system was evaluated.

The results of the CCK assay indicated that degenerated NPCs in the co-culture system exhibited a greater level of proliferation than those in the NPC mono-culture. Certain studies have revealed that stem cells may promote the proliferation of other cells $(26,31)$. This may be explained by the finding that stem cells secrete various growth factors, chemokines and cytokines, which have paracrine and autocrine activities, and these secreted bioactive factors suppress the local immune system, inhibit fibrosis (scar formation) and apoptosis, enhance angiogenesis, and stimulate mitosis and differentiation of tissue-intrinsic reparative or stem cells (18). Umeda et al (32) suggested that bone marrow cells are effective for increasing the proliferative and matrix synthesis capacity of NPCs. It is known that telomerase is induced in a primitive subset of progenitor cells and is downregulated upon further proliferation and differentiation of these cells (33). This provided an explanation as to why the telomerase activity level of NPCs in the co-culture system was higher than that in the mono-culture group, as it was demonstrated that stem cell activity enhanced the viability of NPCs. Nelson and Chen (34) proposed the theory that cell-cell signaling by direct contact increases cell proliferation via phosphoinositide 3-kinase-dependent signaling. The co-culture may promote the proliferation, viability and phenotypic expression of cells by cell signaling pathways and the expression of specific adhesion molecules. This results in cell proliferation and an increase in cell viability, which was consistent with the results regarding the GAG and DNA content. The above results indicated that ADSCs may be ideal cells in the treatment of IVD degeneration, and the co-culture of NPCs with ADSCs significantly enhanced the biological activity of NPCs, including the enhancement of cell proliferation, DNA synthesis and GAG content.

In addition, ADSCs also affected the differentiation of NPCs. NPCs have a chondrocyte-like appearance and express marked levels of Sox9, type II collagen mRNA and aggrecan, appearing rounded and enclosed within a lacuna (35). However, degenerated NPCs lose the chondrocyte sub-type with the downregulation of chondrocyte-associated genes. It has been reported that BMSCs are able to promote the restoration of the 
lost chondrocyte sub-type in de-differentiated chondrocytes Therefore, the chondrogenic differentiation of degenerated NPCs was evaluated in the co-culture system, and the results were consistent with those of previous studies (36). The expression of SOX-9, collagen type II and aggrecan in the co-culture group was higher than that in the control group at different time-points. The differentiation of NPCs was determined by ADSCs co-culture system, and TGF- $\beta 1$ and IGF- 1 , which are considered major regulatory cytokines, secreted by ADSCs, are able to promote the chondrogenic differentiation of degenerated NPCs. Therefore, the levels of growth factors in the supernatant were assessed in the two groups, and the results showed that the growth factors secreted by ADSCs were present in the co-culture medium and therefore had an important role in the chondrogenic differentiation of NPCs. Sun et al (37) demonstrated that direct co-cultures of ADSCs and NP cells stimulated ADSCs differentiation into the NP cell phenotype.

In conclusion, the present study confirmed that co-culture of ADSCs and NPCs not only resulted in the increased proliferation and viability of NPCs, but also promoted the expression of type II collagen, SOX-9 and aggrecan genes, indicating that the injection of ADSCs into degenerative IVD may be a feasible and promising therapy in the treatment of IVD disorders.

\section{Acknowledgements}

This study was supported by the National Natural Science Foundation of China (grant no. 30872607).

\section{References}

1. Adams MA, Lama P, Zehra U and Dolan P: Why do some intervertebral discs degenerate, when others (in the same spine) do not? Clin Anat 28: 195-204, 2015.

2. Ohtori S, Inoue G, Miyagi M and Takahashi K: Pathomechanisms of discogenic low back pain in humans and animal models. Spine J pi (Suppl): S1529-S9430, 2014.

3. Parker SL, Godil SS, Mendenhall SK, Zuckerman SL, Shau DN and McGirt MJ: Two-year comprehensive medical management of degenerative lumbar spine disease (lumbar spondylolisthesis, stenosis, or disc herniation): a value analysis of cost, pain, disability and quality of life: clinical article. J Neurosurg Spine 21: 143-149, 2014.

4. Kim SH, Song JE, Lee D and Khang G: Demineralized bone particle impregnated poly (1-Lactide-co-Glycolide) scaffold for application in tissue-engineered intervertebral discs. J Biomater Sci Polym Ed Nov 29, 2011 (Epub ahead of print).

5. Bron JL, Helder MN, Meisel HJ, Van Royen BJ and Smit TH: Repair, regenerative and supportive therapies of the annulus fibrosus: achievements and challenges. Eur Spine J 18: 301-313, 2009.

6. Tow BP, Hsu WK and Wang JC: Disc regeneration: a glimpse of the future. Clin Neurosurg 54: 122-128, 2007.

7. Thacher C: Neuroanatomic and pathophysiologic aspects of intervertebral disc disease in the dog. Probl Vet Med 1: 337-357, 1989.

8. Jezussek D, Schuh A, Hönle W and Janka M: Conservative therapeutic options in intervertebral disc disease. MMW Fortschr Med 152: 36-39, 2010.

9. Sakai D: Future perspectives of cell-based therapy for intervertebral disc disease. Eur Spine J 17 (Suppl 4): 452-458, 2008.

10. Johnson WE and Roberts S: 'Rumours of my death may have been greatly exaggerated': a brief review of cell death in human intervertebral disc disease and implications for cell transplantation therapy. Biochem Soc Trans 35: 680-682, 2007.

11. Sakai D, Nakamura Y, Nakai T, et al: Exhaustion of nucleus pulposus progenitor cells with ageing and degeneration of the intervertebral disc. Nat Commun 3: 1264, 2012.

12. Gruber HE, Johnson TL, Leslie K, et al: Autologous intervertebral disc cell implantation: a model using Psammomys obesus, the sand rat. Spine (Phila Pa 1976) 27: 1626-1633, 2002.
13. Okuma M, Mochida J, Nishimura K, Sakabe K and Seiki K: Reinsertion of stimulated nucleus pulposus cells retards intervertebral disc degeneration: an in vitro and in vivo experimental study. J Orthop Res 18: 988-997, 2000

14. Martinez-Morales PL, Revilla A, Ocaña I, et al: Progress in stem cell therapy for major human neurological disorders. Stem Cell Rev 9: 685-699, 2013.

15. Wang J, Liao L, Wang S and Tan J: Cell therapy with autologous mesenchymal stem cells-how the disease process impacts clinical considerations. Cytotherapy 15: 893-904, 2013.

16. Wagner W, Wein F, Seckinger A, et al: Comparative characteristics of mesenchymal stem cells from human bone marrow, adipose tissue and umbilical cord blood. Exp Hematol 33: 1402-1416, 2005.

17. Kilroy GE, Foster SJ, Wu X, et al: Cytokine profile of human adipose-derived stem cells: expression of angiogenic, hematopoietic and pro-inflammatory factors. J Cell Physiol 212: 702-709, 2007.

18. Caplan AI and Dennis JE: Mesenchymal stem cells as trophic mediators. J Cell Biochem 98: 1076-1084, 2006.

19. Boomsma RA and Geenen DL: Mesenchymal stem cells secrete multiple cytokines that promote angiogenesis and have contrasting effects on chemotaxis and apoptosis. PLoS One 7: e35685, 2012.

20. Mwale F, Wang HT, Roughly P, Antoniou J and Haglund L: Link $\mathrm{N}$ and mesenchymal stem cells can induce regeneration of the early degenerate intervertebral disc. Tissue Eng Part A 20: 2942-2949, 2014.

21. Yamamoto Y, Mochida J, Sakai D, et al: Upregulation of the viability of nucleus pulposus cells by bone marrow-derived stromal cells: significance of direct cell-to-cell contact in coculture system. Spine (phila pa 1976) 29: 1508-1514, 2004.

22. Wei A, Chung SA, Tao H, et al: Differentiation of rodent bone marrow mesenchymal stem cells into intervertebral disc-like cells following coculture with rat disc tissue. Tissue Eng Part A 15: 2581-2595, 2009.

23. Sun Z, Luo B, Liu ZH, Samartzis D, Liu Z, Gao B, Huang L and Luo ZJ: Adipose-derived stromal cells protect intervertebral disc cells in compression: Implications for stem cell regenerative disc therapy. Int J Biol Sci 11: 133-143, 2015.

24. Lu ZF, Zandieh Doulabi B, Wuisman PI, Bank RA and Helder MN: Differentiation of adipose stem cells by nucleus pulposus cells: configuration effect. Biochem Biophys Res Commun 359: 991-996, 2007.

25. Kim JY, Choeng HC, Ahn C and Cho SH: Early and late changes of MMP-2 and MMP-9 in bleomycin-induced pulmonary fibrosis. Yonsei Med J 50: 68-77, 2009.

26. Niu CC, Yuan LJ, Lin SS, Chen LH and Chen WJ: Mesenchymal stem cell and nucleus pulposus cell coculture modulates cell profile. Clin Orthop Relat Res 467: 3263-3272, 2009.

27. Schmidmaier G, Herrmann S, Green J, et al: Quantitative assessment of growth factors in reaming aspirate, iliac crest and platelet preparation. Bone 39: 1156-1163, 2006.

28. Hass R, Kasper C, Böhm S and Jacobs R: Different populations and sources of human mesenchymal stem cells (MSC): A comparison of adult and neonatal tissue-derived MSC. Cell Commun Signal 9: 12,2011

29. Pittenger MF, Mackay AM, Beck SC, et al: Multilineage potential of adult human mesenchymal stem cells. Science 284: 143-147, 1999.

30. Sobajima S, Vadala G, Shimer A, Kim JS, Gilbertson LG and Kang JD: Feasibility of a stem cell therapy for intervertebral disc degeneration. Spine J 8: 888-896, 2008.

31. Watanabe T, Sakai D, Yamamoto Y, et al: Human nucleus pulposus cells significantly enhanced biological properties in a coculture system with direct cell-to-cell contact with autologous mesenchymal stem cells. J Orthop Res 28: 623-630, 2010.

32. Umeda M, Kushida T, Sasai K, et al: Activation of rat nucleus pulposus cells by coculture with whole bone marrow cells collected by the perfusion method. J Orthop Res 27: 222-228, 2009.

33. Chiu CP, Dragowska W, Kim NW, et al: Differential expression of telomerase activity in hematopoietic progenitors from adult human bone marrow. Stem Cells 14: 239-248, 1996.

34. Nelson CM and Chen CS: Cell-cell signaling by direct contact increases cell proliferation via a PI3K-dependent signal. FEBS Lett 514: 238-242, 2002.

35. Sive JI, Baird P, Jeziorsk M, Watkins A, Hoyland JA and Freemont AJ: Expression of chondrocyte markers by cells of normal and degenerate intervertebral discs. Mol Pathol 55: 91-97, 2002.

36. Maidhof R, Alipui DO, Rafiuddin A, Levine M, Grande DA, and Chahine NO: Emerging trends in biological therapy for intervertebral disc degeneration. Discov Med 14: 401-411, 2012.

37. Sun Z, Liu ZH, Zhao XH, Sun L, Chen YF, Zhang WL, Gao Y, Zhang YZ, Wan ZY, Samartzis D, Wang HQ and Luo ZJ. Impact of direct cell co-cultures on human adipose-derived stromal cells and nucleus pulposuscells. J Orthop Res 31): 1804-1813, 2013. 